\title{
Severe carbon monoxide poisoning from waterpipe smoking: a public health concern
}

\begin{abstract}
We believe this is the first Australian report of severe carbon monoxide poisoning caused by waterpipe use. Carbon monoxide poisoning causes neurological dysfunction and myocardial toxicity, effects that can be irreversible. Despite a widespread misconception that waterpipes are safer than cigarettes, the recognised risks of tobacco products also apply to waterpipe use.
\end{abstract}

\section{Clinical record}

A 20-year-old woman was brought by ambulance to the emergency department of a district hospital after an episode of presyncope. She described symptoms of severe light-headedness, mild headache and nausea, but denied experiencing weakness or sensory disturbances. Seizure activity was not reported. She had used a waterpipe for 1 hour before the onset of symptoms. Although this was her first hospital presentation with these symptoms, she had frequently experienced queasiness and light-headedness after using a waterpipe, which she did on most days of the week, each session lasting about 45 to 60 minutes. She denied depression, suicidal ideation, and recent alcohol or drug use.

The patient appeared lethargic. Her vital signs were within normal limits: blood pressure, $115 / 70 \mathrm{mmHg}$; pulse rate, 75 beats $/ \mathrm{min}$; temperature, $36.7^{\circ} \mathrm{C}$; $\mathrm{Spo}_{2}, 98 \%$; and Glasgow Coma Scale score, 15 . Her pupils were dilated $(5 \mathrm{~mm})$, equal and reactive to light. Her cranial nerve, upper limb and lower limb functions were normal. There was no nystagmus, nor did she have any cerebellar symptoms. Her chest was clear and her heart sounds were normal, with no murmurs. Full blood count and biochemistry and urinalysis data were normal. Severe carbon monoxide $(\mathrm{CO})$ poisoning was diagnosed on the basis of the initial venous and arterial blood gas levels on room air (Box 1). The results of a urinary drug screen and blood alcohol testing were negative.

High-flow oxygen was administered to the patient, and she was transferred to our institution. The results of serial

Rajesh N Subbiah MBBS, PhD, FRACP1,2,

\section{Cameron J Holloway} DPhil, FRACP, FCSANZ1,2,3

$$
\begin{array}{r}
\text { 1St Vincent's Hospital, } \\
\text { Sydney, NSW. } \\
\text { 2 St Vincent's Clinical School, } \\
\text { University of } \\
\text { New South Wales, Sydney, } \\
\text { NSW. } \\
\text { 3 Victor Chang Cardiac } \\
\text { Research Institute, } \\
\text { Sydney, NSW. } \\
\text { 4 Lowy Cancer Research } \\
\text { Centre, University of } \\
\text { New South Wales, } \\
\text { Sydney, NSW. } \\
\text { louis.wang@ } \\
\text { unsw.edu.au }
\end{array}
$$

doi: 10.5694/mjal4.01264

1 Initial venous and arterial blood gas levels of the patient while breathing room air after using a waterpipe for 1 hour* troponin $\mathrm{T}$ assessment 3 and 14 hours after presentation were negative $(<3 \mathrm{ng} / \mathrm{L})$. Her initial electrocardiogram (ECG) showed a normal sinus rhythm. T wave inversion was noted in the anteroseptal leads 5 hours after presentation, but had normalised 9 hours after presentation (Appendix 2). Transthoracic echocardiography showed that left and right ventricular size and systolic function were normal, with normal left ventricular wall thickness, no regional wall motion abnormalities, and normal valve function. Computed tomography coronary angiography was performed because of dynamic $T$ wave changes referable to the left anterior descending artery, but showed that the coronary arteries were normal. The patient was observed for 24 hours and discharged the next day, and was advised to abstain from waterpipe use in the future.

\section{Discussion}

We believe this is the first report in Australia of severe CO poisoning caused by waterpipe use. A waterpipe, also known as a hookah, narghile, shisha or goza, is an apparatus for smoking organic material (often flavoured or non-flavoured tobacco, although non-tobacco herbal preparations are also available). Smoke formed by the heating of organic material is siphoned through water before being inhaled. Variants of the waterpipe have been used in the Middle East, Africa and Asia since the 16th century, and were also popular in Victorian England. Alice encountered the Caterpillar smoking a hookah on a mushroom in Alice's adventures in Wonderland (Box 2). ${ }^{1}$ The portrayal by Lewis Carroll of the lethargic, irritable and forgetful Caterpillar in this classic children's tale afforded an astonishingly accurate depiction of the dangerous physiological effects of waterpipe use.

Although longstanding public health initiatives have reduced overall rates of cigarette smoking, waterpipe use, especially by young adults, poses an important public health threat. Despite concerns about the rapid increase in waterpipe use across the world (prevalence of $6 \%-34 \%$ among Middle Eastern adolescents and 5\%-17\% among American adolescents), ${ }^{2}$ its prevalence in Australia is unclear. The only available study, a survey of 1102 Arabicspeaking residents in south-west Sydney, found that $11.4 \%$ reported current use. ${ }^{3}$

Two main factors have contributed to its popularity: the pleasant experience of social pipe smoking, and the ongoing misconception that the passage of smoke through water before inhalation filters out most toxic substances. 


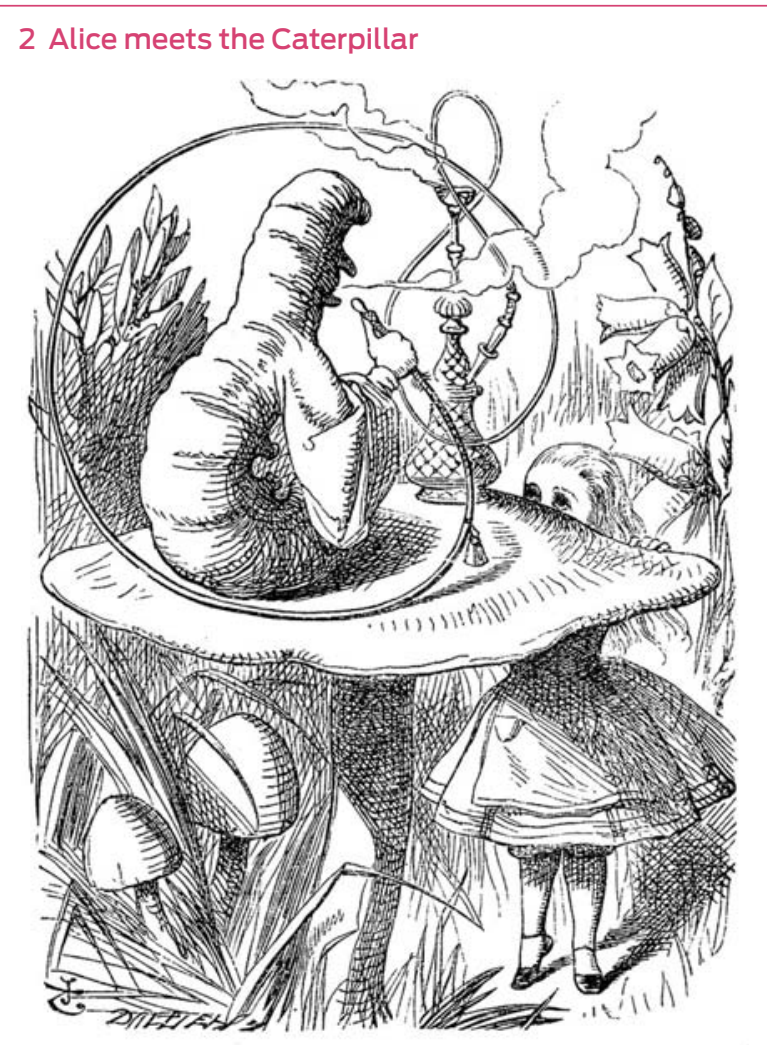

From Alice's adventures in Wonderland by Lewis Carroll. Illustration by Sir John Tenniel, 1865.

This erroneous perception of reduced harm when compared with cigarette smoking is reinforced by the availability of herbal (tobacco-free) preparations. Waterpipe users, however, typically inhale greater amounts of smoke than cigarette smokers. One session of waterpipe use exposes the user to the same amount of smoke as 50-100 cigarettes. ${ }^{4,5}$ As a result, waterpipe users are subject to similar risks of cancer, heart disease, respiratory illness, pregnancy complications and other smoking-related health problems.

$\mathrm{CO}$ poisoning is also a genuine risk for users, with $\mathrm{CO}$ exposure during waterpipe use being almost nine times higher than for cigarette smoking, and peak carboxyhaemoglobin levels three times higher. ${ }^{5}$ Passive exposure to $\mathrm{CO}$ also occurs in hookah lounges, and should be of concern to pregnant women, as fetuses are particularly sensitive to low levels of CO. During waterpipe use, CO is formed from incomplete combustion of charcoal and organic material owing to the reduced oxygen content within the waterpipe apparatus and the relatively low heating temperature. CO binds to haemoglobin with high affinity (more than 200 times that of oxygen) to form carboxyhaemoglobin, which shifts the oxyhaemoglobin dissociation curve markedly to the left, impairing oxygen delivery throughout the body. ${ }^{6}$ Due to the high affinity of CO for haemoglobin, significant carboxyhaemoglobinaemia can develop during even relatively low-level $\mathrm{CO}$ exposure. This reduced oxygen-carrying capacity is not apparent, however, in the reported values for oxygen saturation of haemoglobin (Appendix 1), as they are based on the haemoglobin fraction that can carry oxygen; the carboxyhaemoglobin fraction is thus excluded from the calculation of oxygen saturation.

CO poisoning causes myocardial toxicity. Transient ECG abnormalities, suggestive of myocardial ischaemia, are relatively common, and are evident in $30 \%$ of patients with moderate to severe $\mathrm{CO}$ poisoning. ${ }^{7}$ This occurs even in the absence of significant coronary artery disease, and probably reflects impaired cellular respiration. $\mathrm{CO}$ also acts as an important signalling molecule, capable of altering the function of various cellular ion channels important to the cardiac action potential, as well as of proteins that regulate intracellular calcium levels in cardiomyocytes. ${ }^{8} \mathrm{CO}$ binding to myoglobin may also account for impaired left ventricular systolic function, ${ }^{9}$ with the degree of dysfunction correlated with carboxyhaemoglobin levels and duration of CO exposure. ${ }^{10}$ Left ventricular dysfunction is usually transient, with recovery occurring within 24 hours of $\mathrm{CO}$ poisoning. This may explain the normal left ventricular function seen in this patient's ECG, performed 19 hours after presentation. Myocardial fibrosis can develop, however, after a single episode of severe CO poisoning. ${ }^{11}$ Neurological symptoms of CO toxicity include headache, dizziness, weakness, nausea, confusion and anterograde amnesia. In the longer term, CO poisoning can also cause lesions in the basal ganglia. Treatment of $\mathrm{CO}$ poisoning is largely supportive and involves administration of high-flow oxygen to hasten the elimination of $\mathrm{CO}$. In severe cases, and where facilities are available, hyperbaric oxygen therapy can be considered.

The World Health Organization Framework Convention on Tobacco Control (2005) recommended better regulation of waterpipe use, including health warnings on packaging, prohibiting claims of harm reduction and safety, and banning it in public places, similar to measures currently in place for cigarette smoking. ${ }^{4}$ In many countries, including Australia, waterpipe smoking has previously escaped the strict regulation imposed on other tobacco products, particularly as tobacco is not always the major constituent of hookah preparations. Although harm minimisation techniques, such as improving ventilation in hookah lounges and limiting the duration of smoking sessions, may reduce the risk of severe $\mathrm{CO}$ poisoning, public health measures should focus on encouraging changes to existing legislation, and on discouraging waterpipe use, especially among young adults, by highlighting the health risks.

Acknowledgements: Louis Wang is a recipient of a National Health and Medical Research Council, National Heart Foundation of Australia and Royal Australasian College of Physicians Postgraduate Scholarship. Cameron Holloway is a recipient of a National Heart Foundation of Australia NSW Cardiovascular Research Network Life Sciences Fellowship.

Competing interests: No relevant disclosures.

Provenance: Not commissioned; externally peer reviewed.

References are available online at www.mja.com.au. 
1 Carroll L. Alice's adventures in Wonderland. London: Macmillan, 1865.

2 Maziak W. The global epidemic of waterpipe smoking. Addict Behav 2011; 36: 1-5.

3 Carroll T, Poder N, Perusco A. Is concern about waterpipe tobacco smoking warranted? Aust N Z J Public Health 2008; 32: 181-182.

4 WHO Study Group on Tobacco Product Regulation. Advisory note: waterpipe tobacco smoking: health effects, research needs and recommended actions by regulators. Geneva: World Health Organization, 2005. http://www.who.int/ tobacco/global_interaction/tobreg/Waterpipe\%20 recommendation_Final.pdf (accessed Oct 2014).

5 Eissenberg T, Shihadeh A. Waterpipe tobacco and cigarette smoking. Am J Prev Med 2009; 37: 518-523.

6 Weaver LK. Carbon monoxide poisoning. N Engl J Med 2009; 360: 1217-1225.

7 Satran D, Henry CR, Adkinson C, et al. Cardiovascular manifestations of moderate to severe carbon monoxide poisoning. J Am Coll Cardiol 2005; 45: 1513-1516.

8 Peers C, Steele DS. Carbon monoxide: a vital signalling molecule and potent toxin in the myocardium. J Mol Cell Cardiol 2012; 52: 359-365.

9 Cramlet SH, Erickson HH, Gorman HA. Ventricular function following acute carbon monoxide exposure. J Appl Physiol 1975; 39: 482-486.

10 Kalay N, Ozdogru I, Cetinkaya Y, et al. Cardiovascular effects of carbon monoxide poisoning. Am J Cardiol 2007; 99: 322-324.

1 Henry TD, Lesser JR, Satran D. Myocardial fibrosis from severe carbon monoxide poisoning detected by cardiac magnetic resonance imaging. Circulation 2008; 118: 792. 